\title{
Extension of Delta-Wye and Wye-Delta Conversions Applicable to Electric Circuits with Voltage Sources
}

\author{
Je-Young Choi
}

\author{
Department of Smart IT, U1 University, Eumbong, Asan - 31415, Korea; jychoi@u1.ac.kr
}

\section{Abstract}

Objectives: To generalize the conventional delta-wye and wye-delta conversions. Methods/Statistical Analysis: The delta-wye and wye-delta conversions have been used to simplify complex electric circuits (networks) consisting of resistors. Three terminal networks consisting of resistors and voltage sources in a wye configuration and in a delta configuration are compared. Findings: In this paper we obtained the conversion relations for emfs from the delta configuation to the wye configuration and vice versa. As an example of the generalization, the conversions are applied to a Wheatstone-bridge type circuit of five batteries and five resistors to obtain its Thévenin's equivalent. The conversions are applicable to ac as well as dc circuits. As an example of ac circuits the currents through unbalanced loads in a wye configuration connected to a three-phase generator in a delta configuration are calculated. Application/Improvements: The generalized conversions allow to simplify dc and ac circuits.

Keywords: Delta-Wye Conversion, Electric Circuit, Voltage Source, Wye-Delta Conversion

\section{Introduction}

The Thévenin's theorem states that any two-terminal electric circuit of voltage sources (ideal batteries for dc circuits) and resistors is equivalent to a voltage source and a resistor in series (Thévenin's equivalent). The equivalent emf is given by the open-circuit voltage between the output terminals. The equivalent resistance is obtained by replacing all the sources by short circuits and calculating the resistance between the output terminals. 1

A series-parallel circuit which is formed by combining series and/or parallel combinations can be reduced by successively replacing each series or parallel combination (series/parallel reduction) with an equivalent source and an equivalent resistor in series. $\frac{1,2}{2}$ To be precise, let us introduce a column vector $(E, R)^{T}$ for a pair of a voltage source with emf $E$ and a resistor with resistance $R$ connected in series. Hence a battery with emf $E$ and an internal resistance $r$ is described by a column vector $(E, r)^{T}$ while a pure resistor with resistance $R$ by a column vector $(0, R)^{T}$. Then the Thévenin's equivalent of two pairs $\left(E_{1}\right.$, $\left.R_{1}\right)^{T}$ and $\left(E_{2}, R_{2}\right)^{T}$ in series is given by

$$
\left(\begin{array}{c}
E_{e q} \\
R_{e q}
\end{array}\right)=\left(\begin{array}{l}
E_{1} \\
R_{1}
\end{array}\right)+\left(\begin{array}{l}
E_{2} \\
R_{2}
\end{array}\right)=\left(\begin{array}{c}
E_{1}+E_{2} \\
R_{1}+R_{2}
\end{array}\right)
$$

and in parallel by

$$
\left(\begin{array}{c}
E_{e q} \\
R_{e q}
\end{array}\right) \equiv\left(\begin{array}{l}
E_{1} \\
R_{1}
\end{array}\right) \|\left(\begin{array}{c}
E_{2} \\
R_{2}
\end{array}\right),
$$

where

$$
\begin{aligned}
& R_{e q}=\frac{R_{1} R_{2}}{R_{1}+R_{2}}, \\
& E_{e q}=\frac{R_{2} E_{1}+R_{1} E_{2}}{R_{1}+R_{2}} .
\end{aligned}
$$

Equations (3) and (4) for the parallel combination are called the Millman's theorem. ${ }^{1}$ The operation $\|$ for the parallel combination is commutative and associative as the addition for the series combination is.

On the contrary, a complex circuit which does not belong to series-parallel circuits requires Kirchhoff's rules or loop analysis to obtain its equivalent emf and 
equivalent resistance. ${ }^{1}$ Baak described how to exploit a variational principle instead of using Kirchhoff's voltage rule. ${ }^{-}$Electric circuits can also be solved by using random walks. ${ }^{4}$ However, all these methods have nonlocality in character which requires considering a circuit as a whole.

An alternative method is to use delta-wye $(\Delta-Y)$ conversion (also referred to as triangle-star or $\Pi-\mathrm{T}$ conversion) or its inverse, wye-delta (Y- $\Delta$ ) conversion (also referred to as star-triangle or T- $\Pi$ conversion) for subcircuits of resistors with three terminals..,$\frac{15}{}$ Both conversions are local by construction. A $\Delta-\mathrm{Y}$ conversion eliminates a loop but adds a node by transforming a $\Delta$ configuration into a $\mathrm{Y}$ configuration while a $\mathrm{Y}-\Delta$ conversion eliminates a node but adds a loop by transforming a $\mathrm{Y}$ configuration into a $\Delta$ configuration. Complex circuits such as shown in Figure 1(a) and in Figure 1(b) can be transformed into series-parallel circuits by applying $\Delta-Y$ conversions three times, and by applying $\Delta-Y$ conversions nine times and $\mathrm{Y}-\Delta$ conversions four times, respectively.

In exactly solvable statistical models there is a similar construct, called the star-triangle relation, which transforms a $\mathrm{Y}$ (star) configuration of spin interactions into a $\Delta$ (triangle) configuration of spin interactions and vice versa, and can determine the solvability condition from which the exact critical temperature and the exact partition function can be obtained. $\underline{6}$

In this paper the $\Delta-\mathrm{Y}$ and $\mathrm{Y}-\Delta$ conversions are extended so that they are applicable to circuits consisting of resistors and voltage sources. A possible origin of voltage sources in series with resistors is from thermal noise due to thermal agitation of conduction electrons in conductors so that each resistor can be modeled to have a minute random voltage source in series. $\frac{7.8}{7}$ The simplest complex circuit is the unbalanced Wheatstone bridge of five resistors. The extension is exemplified with the Wheatstone-bridge type circuit of five batteries and five resistors considered recently. ${ }^{9}$ It is noted that the conversions are also applicable to ac circuits where the resistors and ideal batteries are replaced with passive elements and ac voltage sources, respectively. Finally the conversions are applied to three-phase systems.

\section{2. $\Delta-Y$ and $Y-\Delta$ Conversions}

In Figure 2, $\Delta$ and $\mathrm{Y}$ configurations between terminals $N_{1}, N_{2}$, and $N_{3}$ are depicted, where each branch contains a voltage source with emf $E_{\mathrm{i}}$ and a resistor with resistance $R_{\mathrm{i}}$ in series, which is referred to as branch $i$ from now on.
In the case of a real battery as a voltage source its internal resistance is included in the associated resistance. We want to find the values $E_{\mathrm{a}}, R_{\mathrm{a}}, E_{\mathrm{b}}, R_{\mathrm{b}}, E_{\mathrm{c}}$ and $R_{\mathrm{c}}$ of the equivalent $Y$ configuration from the given $E_{\alpha}, R_{\alpha}, E_{\beta}, R_{\beta}, E_{\gamma}$ and $R_{\gamma}$ of the $\Delta$ configuration and vice versa.

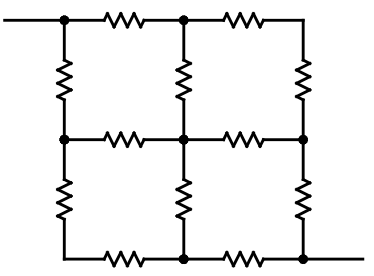

(a)

(b)

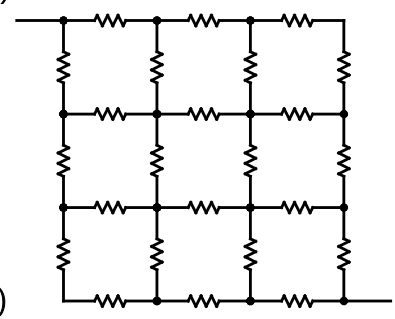

Figure 1. Examples of complex circuits of resistors with two terminals.

Applying the Thévenin's theorem to the terminals $N_{1}$ and $N_{2}$, we get the equivalent resistance $R_{2,1}$ and the equivalent emf $E_{2,1}$

$$
\left(\begin{array}{c}
E_{2,1} \\
R_{2,1}
\end{array}\right)=\left(\begin{array}{c}
-E_{\gamma} \\
R_{\gamma}
\end{array}\right) \|\left[\left(\begin{array}{c}
E_{\alpha} \\
R_{\alpha}
\end{array}\right)+\left(\begin{array}{c}
E_{\beta} \\
R_{\beta}
\end{array}\right)\right]
$$

for the $\Delta$ configuration and

$$
\left(\begin{array}{l}
E_{2,1} \\
R_{2,1}
\end{array}\right)=\left(\begin{array}{c}
E_{a} \\
R_{a}
\end{array}\right)+\left(\begin{array}{c}
-E_{b} \\
R_{b}
\end{array}\right)=\left(\begin{array}{c}
E_{a}-E_{b} \\
R_{a}+R_{b}
\end{array}\right)
$$

for the $\mathrm{Y}$ configuration. The minus signs in front of some emfs appear because the directions of the corresponding batteries in Figure 2 are opposite to the chosen directions of traversal. Equating Equations (5) and (6) and using Equations (1)-(4) gives

$$
\begin{gathered}
R_{a}+R_{b}=\frac{\left(R_{\alpha}+R_{\beta}\right) R_{\gamma}}{R_{\alpha}+R_{\beta}+R_{\gamma}}, \\
E_{a}-E_{b}=\frac{R_{\gamma}\left(E_{\alpha}+E_{\beta}\right)-\left(R_{\alpha}+R_{\beta}\right) E_{\gamma}}{R_{\alpha}+R_{\beta}+R_{\gamma}} .
\end{gathered}
$$

Similarly for $N_{2}-N_{3}$,

$$
R_{b}+R_{c}=\frac{\left(R_{\beta}+R_{\gamma}\right) R_{\alpha}}{R_{\alpha}+R_{\beta}+R_{\gamma}}
$$




$$
E_{b}-E_{c}=\frac{R_{\alpha}\left(E_{\beta}+E_{\gamma}\right)-\left(R_{\beta}+R_{\gamma}\right) E_{\alpha}}{R_{\alpha}+R_{\beta}+R_{\gamma}}
$$

and for $N_{3}-N_{1}$,

$$
\begin{gathered}
R_{c}+R_{a}=\frac{\left(R_{\gamma}+R_{\alpha}\right) R_{\beta}}{R_{\alpha}+R_{\beta}+R_{\gamma}}, \\
E_{c}-E_{a}=\frac{R_{\beta}\left(E_{\gamma}+E_{\alpha}\right)-\left(R_{\gamma}+R_{\alpha}\right) E_{\beta}}{R_{\alpha}+R_{\beta}+R_{\gamma}} .
\end{gathered}
$$

Adding Equations (7), (9), and (11) and dividing the result by two yields

$$
R_{a}+R_{b}+R_{c}=\frac{R_{\alpha} R_{\beta}+R_{\beta} R_{\gamma}+R_{\gamma} R_{\alpha}}{R_{\alpha}+R_{\beta}+R_{\gamma}} .
$$

Subtracting Equations (9), (11), and (7) from equation (13) gives the well-known conversion relations for resistances, $\stackrel{-1}{-}$ respectively,

$$
\begin{aligned}
& R_{a}=\frac{R_{\beta} R_{\gamma}}{R_{\alpha}+R_{\beta}+R_{\gamma}}, \\
& R_{b}=\frac{R_{\gamma} R_{\alpha}}{R_{\alpha}+R_{\beta}+R_{\gamma}}, \\
& R_{c}=\frac{R_{\alpha} R_{\beta}}{R_{\alpha}+R_{\beta}+R_{\gamma}} .
\end{aligned}
$$

Only two out of Equations (8), (10) and (12) are independent since all of them add up to zero. Physically the center of the $\mathrm{Y}$ configuration is connected only to the three voltage sources $E_{\mathrm{a}}, E_{\mathrm{b}}$ and $E_{\mathrm{c}}$ so that its voltage cannot be determined and is arbitrary. Thus we choose the cyclic one:

$$
\begin{aligned}
& E_{a}=-\frac{R_{\beta} E_{\gamma}-R_{\gamma} E_{\beta}}{R_{\alpha}+R_{\beta}+R_{\gamma}}+f . \\
& E_{b}=-\frac{R_{\gamma} E_{\alpha}-R_{\alpha} E_{\gamma}}{R_{\alpha}+R_{\beta}+R_{\gamma}}+f, \\
& E_{c}=-\frac{R_{\alpha} E_{\beta}-R_{\beta} E_{\alpha}}{R_{\alpha}+R_{\beta}+R_{\gamma}}+f,
\end{aligned}
$$

where $f$ is an arbitrary constant. Equations (14) and (15) constitute the conversion relations from the $\Delta$ configuration to the $\mathrm{Y}$ configuration. The linearity of equation (15) in $\mathrm{E}_{\alpha}, \mathrm{E}_{\beta}$, and $\mathrm{E}_{\gamma}$ is a consequence of the superposition theorem. ${ }^{1}$ In particular, if $\mathrm{R}_{\alpha}=\mathrm{R}_{\beta}=\mathrm{R}_{\gamma} \equiv 3 \mathrm{R}_{0}$, then equation (15) is simplified: $\mathrm{R}_{\mathrm{a}}=\mathrm{R}_{\mathrm{b}}=\mathrm{R}_{\mathrm{c}}=\mathrm{R}_{0}$ and

$$
\begin{aligned}
& E_{a}=\frac{E_{\beta}-E_{\gamma}}{3}+f, \\
& E_{b}=\frac{E_{\gamma}-E_{\alpha}}{3}+f, \\
& E_{c}=\frac{E_{\alpha}-E_{\beta}}{3}+f .
\end{aligned}
$$

The inverse relations for resistances from the $\mathrm{Y}$ configuration to the $\Delta$ configuration are well known and can be obtained from equation (14): ${ }^{1}$

$$
\begin{aligned}
& R_{\alpha}=\frac{R_{a} R_{b}+R_{b} R_{c}+R_{c} R_{a}}{R_{a}}, \\
& R_{\beta}=\frac{R_{a} R_{b}+R_{b} R_{c}+R_{c} R_{a}}{R_{b}}, \\
& R_{\gamma}=\frac{R_{a} R_{b}+R_{b} R_{c}+R_{c} R_{a}}{R_{c}} .
\end{aligned}
$$

Given $E_{\mathrm{a}}, E_{\mathrm{b}}$, and $E_{\mathrm{c}}$ only two out of $E_{\alpha}, E_{\beta}$ and $E_{\gamma}$ are independent. Equations (10) and (12) can be solved for $E_{\alpha}$ and $E_{\beta}$ to give

$$
\begin{aligned}
& E_{\alpha}=-\left(E_{b}-E_{c}\right)+\frac{R_{c}}{R_{a}}\left(E_{a}-E_{b}+E_{\gamma}\right), \\
& E_{\beta}=-\left(E_{c}-E_{a}\right)+\frac{R_{c}}{R_{b}}\left(E_{a}-E_{b}+E_{\gamma}\right)
\end{aligned}
$$

after equation (17) has been used. By appropriately choosing the expression for $E_{\gamma}$ we can write cyclically

$$
\begin{aligned}
& E_{\alpha}=-\left(E_{b}-E_{c}\right)+\frac{g}{R_{a}}, \\
& E_{\beta}=-\left(E_{c}-E_{a}\right)+\frac{g}{R_{b}}, \\
& E_{\gamma}=-\left(E_{a}-E_{b}\right)+\frac{g}{R_{c}}
\end{aligned}
$$

for some arbitrary constant $g$. Equations (17) and (20) compose the relations for $\mathrm{Y}-\Delta$ conversions.

In order to grasp the meaning of $f$ and $g$ we use Equations (15) and (20) and to write

$$
f=\frac{R_{\alpha} E_{a}+R_{\beta} E_{b}+R_{\gamma} E_{b}}{R_{\alpha}+R_{\beta}+R_{\gamma}},
$$




$$
g=\left(\frac{1}{R_{a}}+\frac{1}{R_{b}}+\frac{1}{R_{c}}\right)^{-1}\left(E_{\alpha}+E_{\beta}+E_{\gamma}\right) .
$$

Equations (14) and (17) are used to write $f$ and $g$ purely in terms of $Y$ and $\Delta$ quantities, respectively:

$$
\begin{aligned}
& f=\left(\frac{1}{R_{a}}+\frac{1}{R_{b}}+\frac{1}{R_{c}}\right)^{-1}\left(\frac{E_{a}}{R_{a}}+\frac{E_{b}}{R_{b}}+\frac{E_{c}}{R_{c}}\right), \\
& g=\frac{R_{\alpha} R_{\beta} R_{\gamma}}{\left(R_{\alpha}+R_{\beta}+R_{\gamma}\right)^{2}}\left(E_{\alpha}+E_{\beta}+E_{\gamma}\right) .
\end{aligned}
$$

It is seen that $f$ is the equivalent emf of the three branches $a, b$ and $c$ in Figure 2(b) in parallel (with terminals $N_{1}$, $N_{2}$, and $N_{3}$ joined) and that $\frac{\left(R_{\alpha}+R_{\beta}+R_{\gamma}\right)^{2}}{R_{\alpha} R_{\beta} R_{\gamma}} g$ is the potential drop around the loop $N_{1} N_{2} N_{3} N_{1}$ due to $R_{\alpha}, R_{\beta}$, and $R_{y}$ in Figure 2(a).

Equations (14) and (17) can be written compactly as

$$
\begin{aligned}
R_{\alpha} R_{a} & =R_{\beta} R_{b}=R_{\gamma} R_{c} \\
& =R_{a} R_{b}+R_{b} R_{c}+R_{c} R_{a} \\
& =\frac{R_{\alpha} R_{\beta} R_{\gamma}}{R_{\alpha}+R_{\beta}+R_{\gamma}} .
\end{aligned}
$$

Using the cross product of vectors, Equations (15) and (20) can also be written as

$$
\begin{aligned}
& \left(E_{a}, E_{b}, E_{c}\right)=-\frac{\left(R_{\alpha}, R_{\beta}, R_{\gamma}\right)}{R_{\alpha}+R_{\beta}+R_{\gamma}} \times\left(E_{\alpha}, E_{\beta}, E_{\gamma}\right)+(1,1,1) f, \\
& \left(E_{\alpha}, E_{\beta}, E_{\gamma}\right)=(1,1,1) \times\left(E_{a}, E_{b}, E_{c}\right)+\left(\frac{1}{R_{a}}, \frac{1}{R_{b}}, \frac{1}{R_{c}}\right) g .
\end{aligned}
$$

Various relations between emfs and resistances of $\Delta$ and $\mathrm{Y}$ configurations can be easily read off from Equations

(25)-(27). For example, dot multiplying equation (26) with $\left(\mathrm{R}_{\alpha}, \mathrm{R}_{\beta}, \mathrm{R}_{\gamma}\right)$ gives equation (21).

\section{Wheatstone-Bridge Type Circuit}

Hong and Choi considered a Wheatstone-bridge type configuration of five batteries where each branch contains a battery with an ideal emf $E_{\mathrm{i}}$ and an internal resistance $R_{\mathrm{i}}{ }^{9}$ But their argument equally applies to the situation depicted in Figure 3(a) where each branch $i$ contains a voltage source (an ideal battery) with an emf $E_{\mathrm{i}}$ and a resistor with the resistance $R_{\mathrm{i}}$ in series. Of course, not every branch has to contain a voltage source.

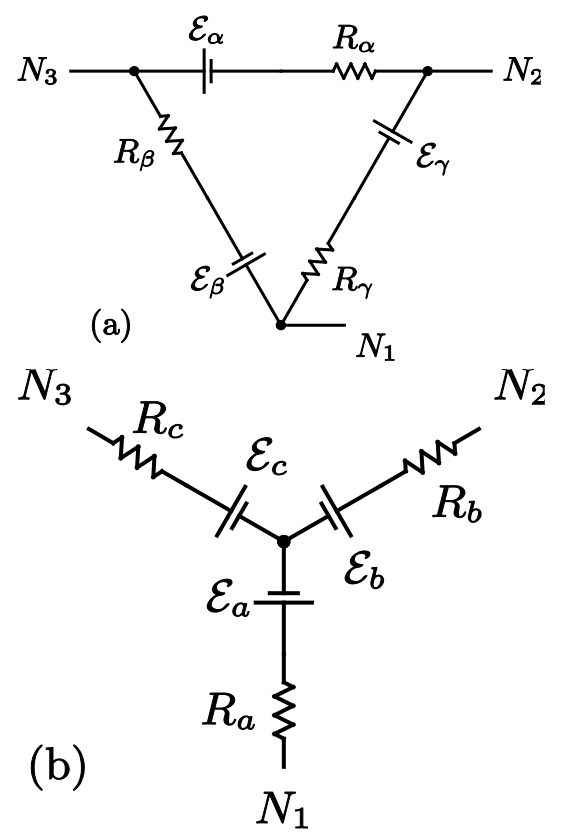

Figure 2. Three-terminal circuits between terminals $N_{1}, N_{2}$, and $N_{3}$ with (a) a $\Delta$ configuration and (b) a Y configuration.

In order to obtain the Thévenin's equivalent the $\Delta$-Y conversion is applied to the left $\Delta$ configuration in Figure 3 (a) consisting of branches 1,2 , and 5 and we identify

$\left(\begin{array}{c}E_{\alpha} \\ R_{\alpha}\end{array}\right)=\left(\begin{array}{l}E_{1} \\ R_{1}\end{array}\right), \quad\left(\begin{array}{c}E_{\beta} \\ R_{\beta}\end{array}\right)=\left(\begin{array}{c}-E_{2} \\ R_{2}\end{array}\right), \quad\left(\begin{array}{l}E_{\gamma} \\ R_{\gamma}\end{array}\right)=\left(\begin{array}{c}E_{5} \\ R_{5}\end{array}\right)$.

In the converted circuit of Figure 3(b), the pair of branches $b$ and 3 and the pair of $a$ and 4 are connected each in series, both of which are connected to each other in parallel and in turn are connected in series to the branch $c$. Hence the Thévenin's equivalent is given by

$$
\left(\begin{array}{c}
E_{e q} \\
R_{e q}
\end{array}\right)=\left(\begin{array}{c}
E_{c} \\
R_{c}
\end{array}\right)+\left[\left(\begin{array}{c}
-E_{b} \\
R_{b}
\end{array}\right)+\left(\begin{array}{c}
E_{3} \\
R_{3}
\end{array}\right)\right] \|\left[\left(\begin{array}{c}
-E_{a} \\
R_{a}
\end{array}\right)+\left(\begin{array}{c}
E_{4} \\
R_{4}
\end{array}\right)\right] .
$$

If we substitute Equations (14) and (15) into equation (29) and use Equations (2) and (28), it is straightforward to show that the equivalent emf $E_{\text {eq }}$ and the equivalent resistance $R_{e q}$ are given by

$$
E_{e q}=A / C, \quad R_{e q}=B / C,
$$




$$
\begin{aligned}
A \equiv & {\left[R_{2}\left(R_{3}+R_{4}\right)+\left(R_{2}+R_{4}\right) R_{5}\right] E_{1}+\left[R_{1}\left(R_{3}+R_{4}\right)+\left(R_{1}+R_{3}\right) R_{5}\right] E_{2} } \\
& +\left[R_{4}\left(R_{1}+R_{2}\right)+\left(R_{2}+R_{4}\right) R_{5}\right] E_{3}+\left[R_{3}\left(R_{1}+R_{2}\right)+\left(R_{1}+R_{3}\right) R_{5}\right] E_{4} \\
& +\left(R_{2} R_{3}-R_{1} R_{4}\right) E_{5}, \\
B \equiv & R_{1} R_{2}\left(R_{3}+R_{4}\right)+\left(R_{1}+R_{2}\right) R_{3} R_{4}+\left(R_{1}+R_{3}\right)\left(R_{2}+R_{4}\right) R_{5}, \\
C \equiv & \left(R_{1}+R_{2}\right)\left(R_{3}+R_{4}\right)+\left(R_{1}+R_{2}+R_{3}+R_{4}\right) R_{5},
\end{aligned}
$$

respectively, as was obtained recently. ${ }^{9}$ In the final result, $f$ in equation (15) is canceled out as expected. Alternatively, the same result can be reached by applying the Y- $\Delta$ conversion to the $Y$ configuration consisting of branches 2,4 , and 5 in Figure 3(a).

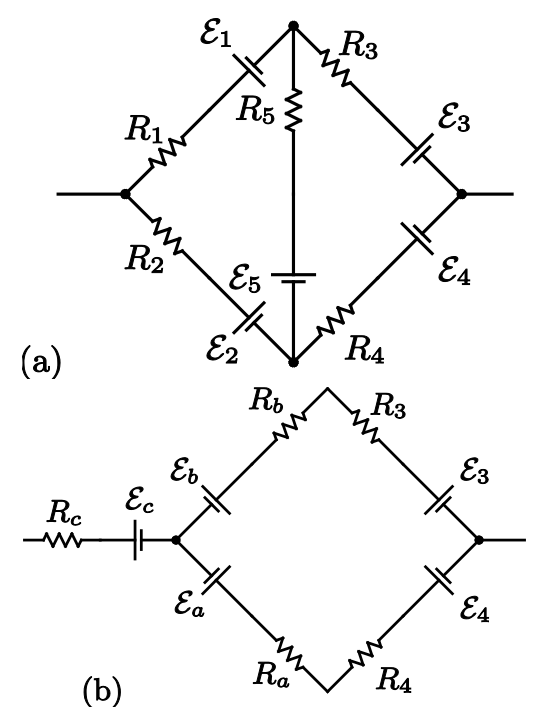

Figure 3. (a) Wheatstone-bridge type combination of five batteries and five resistors. (b) The $\Delta$ configuration in (a) is converted to a $\mathrm{Y}$ configuration.

\section{Three-Phase Systems}

In an ac circuit consisting of ac voltage sources and passive elements like resistors, capacitors, and inductors, the relations developed in section 2 are valid with the substitutions $^{1}$

$$
E_{j} \rightarrow E_{j}=V_{j} e^{i\left(\omega t+\theta_{j}\right)}, \quad R_{j} \rightarrow Z_{j},
$$

where angular frequency $\omega$, phases $\theta_{j}$, and peak voltages $V_{\mathrm{j}}$ take real values and $Z_{\mathrm{j}}$ are complex impedances of passive elements.

Many electric power generators are three-phase because a three-phase power system is more effective than a single-phase in production, transmission, and consumption. Three induction coils in a three-phase generator are arranged $120^{\circ}$ apart with each other, producing three ac voltages which are $120^{\circ}$ out of phase with each other. $\frac{1,10}{10}$
They may be either in a Y configuration or in a $\Delta$ configuration. The three-phase generator is called balanced if peak values of the three voltages are equal in magnitude, exactly $120^{\circ}$ out of phase with each other, and have equal source impedances, and unbalanced otherwise. ${ }^{1}$

(a)

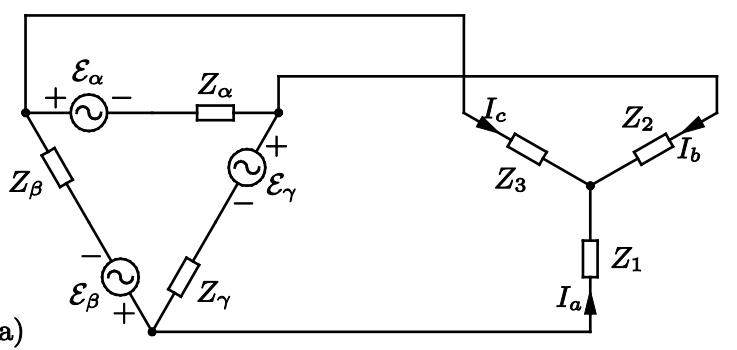

(b)

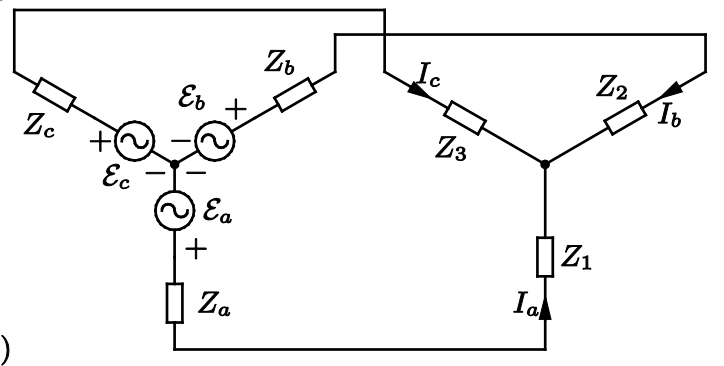

Figure 4. (a) A three-phase generator is connected to unbalanced loads in a $\Delta-Y$ connection. (b) The $\Delta$ configuration of the generator in (a) is converted to a $\mathrm{Y}$ configuration (to make a Y-Y connection).

Let us assume that a balanced three-phase generator consisting of three induction coils modeled with $E_{\alpha}$ and $Z_{\alpha}, E_{\beta}$ and $Z_{\beta}$, and $E_{\gamma}$ and $Z_{\gamma}$ in a $\Delta$ configuration is connected to unbalanced loads $Z_{1}, Z_{2}$ and $Z_{3}$ (that is, $Z_{1}, Z_{2}$ and $Z_{3}$ may be different) in a $Y$ configuration depicted in Figure 4(a), where we include usually neglected impedances $Z_{\alpha}=Z_{\beta}=Z_{\gamma} \equiv 3 Z_{\mathrm{S}}$ of induction coils and

$$
\begin{aligned}
& E_{\alpha}=V e^{i \omega t}, \\
& E_{\beta}=V e^{i\left(\omega t+\frac{2}{3} \pi\right)}, \\
& E_{\gamma}=V e^{i\left(\omega t-\frac{2}{3} \pi\right)}
\end{aligned}
$$

which satisfy the condition

$$
E_{\alpha}+E_{\beta}+E_{\gamma}=0 .
$$

In order to obtain the currents through the loads we convert the $\Delta$ configuration in Figure 4(a) to a Y configuration in Figure 4(b) to make a Y-Y connection (without a neutral line). From Equations (14) and (16) (modified according to equation (34)), we have $Z_{\mathrm{a}}=Z_{\mathrm{b}}=Z_{\mathrm{c}}=Z_{\mathrm{s}}$ and 


$$
\begin{aligned}
& E_{a}=\frac{V}{\sqrt{3}} e^{i\left(\omega t+\frac{1}{2} \pi\right)}, \\
& E_{b}=\frac{V}{\sqrt{3}} e^{i\left(\omega t-\frac{5}{6} \pi\right)}, \\
& E_{c}=\frac{V}{\sqrt{3}} e^{i\left(\omega t-\frac{1}{6} \pi\right)} .
\end{aligned}
$$

where the additive constant $f$ in equation (16) vanishes since we consider phasors.

The current $I_{a}$ through the load $Z_{1}$ can be obtained by noting that the branch containing $E_{b}, Z_{b}$, and $Z_{2}$ and the branch containing $E_{c}, Z_{c}$, and $Z_{3}$ are connected in parallel so that their Thévenin's equivalent is

$$
\left(\begin{array}{c}
E_{e q} \\
Z_{e q}
\end{array}\right)=\left(\begin{array}{c}
E_{b} \\
Z_{b}+Z_{2}
\end{array}\right) \|\left(\begin{array}{c}
E_{c} \\
Z_{c}+Z_{3}
\end{array}\right) .
$$

Hence we have

$$
\begin{aligned}
& I_{a}=\frac{E_{a}-E_{e q}}{Z_{a}+Z_{1}+Z_{e q}} \\
& =\frac{V}{D} e^{i\left(\omega t+\frac{1}{2} \pi\right)}\left(e^{\frac{1}{6} i \pi} Z_{2}+e^{-\frac{1}{6} i \pi} Z_{3}+\sqrt{3} Z_{S}\right),
\end{aligned}
$$

where

$$
D \equiv Z_{1} Z_{2}+Z_{2} Z_{3}+Z_{3} Z_{1}+2\left(Z_{1}+Z_{2}+Z_{3}\right) Z_{S}+3 Z_{S}^{2} .
$$

The currents $I_{b}$ and $I_{c}$ can be obtained similarly:

$$
\begin{aligned}
& I_{b}=\frac{V}{D} e^{i\left(\omega t-\frac{5}{6} \pi\right)}\left(e^{\frac{1}{6} i \pi} Z_{3}+e^{-\frac{1}{6} i \pi} Z_{1}+\sqrt{3} Z_{S}\right), \\
& I_{c}=\frac{V}{D} e^{i\left(\omega t-\frac{1}{6} \pi\right)}\left(e^{\frac{1}{6} i \pi} Z_{1}+e^{-\frac{1}{6} i \pi} Z_{2}+\sqrt{3} Z_{S}\right) .
\end{aligned}
$$

As expected, $I_{a}+I_{b}+I_{c}=0$ satisfying the Kirchhoff's current rule. Due to unbalancing of loads the currents will not be exactly $120^{\circ}$ out of phase with each other. In particular for balanced loads with $Z_{1}=Z_{2}=Z_{3}=Z_{\mathrm{L}}$, the currents take the simple expressions

$$
\begin{aligned}
& I_{a}=\frac{V e^{i\left(\omega t+\frac{1}{2} \pi\right)}}{\sqrt{3}\left(Z_{L}+Z_{S}\right)}, \\
& I_{b}=\frac{V e^{i\left(\omega t-\frac{5}{6} \pi\right)}}{\sqrt{3}\left(Z_{L}+Z_{S}\right)},
\end{aligned}
$$

$$
I_{c}=\frac{V e^{i\left(\omega t-\frac{1}{6} \pi\right)}}{\sqrt{3}\left(Z_{L}+Z_{S}\right)},
$$

which have the three-phase symmetry.

In describing a three-phase generator in a Y configuration, impedances of induction coils are usually ignored (or treated as included in load impedances) so that $Z_{\alpha}=$ $Z_{\beta}=Z_{\gamma}=3 Z_{\mathrm{S}}=0 . \stackrel{11}{ }$ But in an unbalanced three-phase generator with $E_{\alpha}+E_{\beta}+E_{\gamma} \neq 0$, they cannot be neglected in a $\Delta$ configuration since the Kirchhoff's voltage rule around the $\Delta$ loop must vanish. Obviously the above method can be easily applied to unbalanced three-phase generators.

\section{Conclusion}

We generalized $\Delta-\mathrm{Y}$ and $\mathrm{Y}-\Delta$ conversions so that complex circuits consisting of resistors and voltage sources can be simplified and eventually transformed into series-parallel ones by applying these conversions successively. In order to obtain the Thévenin's equivalent of a complex twoterminal circuit it is not necessary to set up simultaneous equations by applying Kirchhoff's rules or loop analysis with taking special care of polarities of sources and directions of currents along resistors with respect to traversal of each loop, but to apply locally the conversion relations to three-terminal subcircuits chosen in the original circuit.

Even though expressions like Equations (31)-(33) look complex, calculations needed to apply $\Delta$-Y and/ or Y- $\Delta$ conversions are systematic and straightforward. Hence the conversions can easily be used in simplifying complex circuits.

The given method is equally applicable to ac circuits where resistances are replaced with complex impedances and voltage sources are oscillating as complex phasors $V_{j}$ $e^{i(\omega t-\theta j)}$ with the same frequency $\omega$ but possibly with different phases $\theta_{j}$. In dealing with three-phase generators, the impedances of induction coils are usually neglected for computational conveniences but cannot be ignored in unbalanced generators in a $\Delta$ configuration. Our extension turns out to provide a simple and easy method to cope with the source impedances.

\section{References}

1. Suresh Kumar KS. Electric Circuits and Networks. 11th ed Dorling Kindersley. New Delhi. 2007.

2. Hong SI, Choi J-Y, Kim B-O. Roles of batteries based on their power production and consumption. New physics. 2004 Mar; 48(3): 223-8. 
3. Van Baak DA. Variational alternatives to Kirchhoff's loop theorem in dc circuits. American Journal of Physics.1999 Jan; 67(1):36-44. Crossref

4. Doyle PG, Snell JL. Random Walks and Electric circuits. Washinton DC: Mathematical Association of America. 1984; Chap 3.

5. Kennelly AE. Equivalence of triangles and stars in conducting circuits. Electrical World and Engineer. 1899; 34:413-4.

6. Baxter RJ. Exactly Solved Models in Statistical Mechanics. London: Academic Press; 1982; 80-6.

7. Johnson JB. Thermal Agitation of Electricity in Conductors. Physical Review. 1928 Jul; 32(1): 97-109. Crossref
8. Nyquist H. Thermal Agitation of Electric Charge in Conductors. Physical Review. 1928 Jul; 32(1):110-3. Crossref

9. Hong SI, Choi J-Y. Structure of equivalent EMF and equivalent internal resistance for a Wheatstone-bridgetype combination of five batteries. New Physics. 2007 Jun; 54(6):526-9.

10. Robertson BL. Three-phase systems with unbalanced wye-connected loads. Electrical Engineering. 1941 Feb; 60(2):80-5. Crossref

11. Youb L. Effects of Unbalanced Voltage on the Steady State of the Induction Motors. International Journal of Electrical Energy. 2014 Mar; 2(1):34-8. Crossref 